\title{
Infrared Open Cavities for Strong Vibrational Coupling
}

\author{
Bar Cohn, Kamalika Das, Arghyadeep Basu, and Lev Chuntonov* \\ Schulich Faculty of Chemistry and Solid State Institute, Technion - Israel Institute of \\ Technology, Haifa 3200003, Israel
}

Supporting Information

\section{Methods}

Fabrication of the antenna arrays

Arrays of half-wavelength gold antennas were fabricated by means of electron beam lithography (EBL) on 1-inch $2 \mathrm{~mm}$-thick $\mathrm{CaF}_{2}$ windows (UV-grade, Crystran, UK). After a thorough cleaning, the windows were coated with hexamethyldisilazne by vapor deposition (Suss MicroTec Delta $82 \mathrm{RC}$ ) for better adhesion. Afterwards, a double-layer negative resist was deposited by spin-coating in two steps (PMMA 495a2 and 950a3), following by baking at $180^{\circ} \mathrm{C}$. For improved conductivity during the EBL writing, a $30 \mathrm{~nm}$-thick layer of Chromium was deposited using electron-beam evaporator (Evatec BAK-501A). The EBL pattern were written on RAITH EBPG 5200. The pattern design involved a $100 \times 100 \mu \mathrm{m}$ sub-lattice, xwhich was duplicated to create a $4 \times 4 \mathrm{~mm}$ array sample. Such approach insured equal exposure of the whole sample area to the electron beam, which was important to avoid stitching imperfections during EBL writing. The exposed pattern was then subjected to a chromium etching solution (Micro Chemicals) followed by a development in a 1:3 methyl isobutylketone-isopropanol mixture. After development, a $5 \mathrm{~nm}$ chromium adhesion and $80 \mathrm{~nm}$ gold layers were deposited using e-beam evaporator. Lastly, a lift-off procedure was conducted with acetone followed by methanol, isopropanol, and water rinsing.

\section{Optical measurements}

The FTIR measurements were performed in the transmission mode on the Thermo-Fischer Nicolet is 10 spectrometer. The sample cell was mounted on an assembly of translational and rotational stages which enabled positioning the array at the focus of the excitation light for various incident angles. A wire-grid polarizer (Thorlabs) was used to control the light polarization. An iris was placed right after the parabolic mirror focusing the light on the sample in order to increase the spatial coherence of the IR Globar source. 
An additional iris was placed just before the collimating parabolic mirror after the sample in order to spatiality resolve the signal emerging from the array. All measurements (except those shown in Figure 4) were conducted in a sample cell constructed with a pair of $2 \mathrm{~mm}$-thick $\mathrm{CaF}_{2}$ windows (one with the fabricated array and another one clean) separated by the $100 \mu \mathrm{m}$ spacer and filled with $\mathrm{CCl}_{4}$ as an indexmatching liquid.

\section{Numerical simulations}

Numerical simulations of the arrays of half-wavelength antennas were conducted with the FDTD method (Lumerical). The antenna dimension used in the simulations was obtained from the corresponding SEM images; the dielectric function of gold was taken from ref. (1); the Lorentz model was used for the dielectric function of PMMA with the parameters from ref. (2).

For boundary conditions in the out-of-plane direction (z-axis), perfectly matched layers (PML) were used, whereas in the in-plane directions (x- and y-axes) we used periodic boundary conditions. For the calculations of dispersion (different incident angles), a Broadband Fixed Angle Source Technique (BFAST) of the plane wave source was used.

The Extinction (1-Transmittance) and Reflection spectra of the arrays were calculated, and the corresponding Absorption spectrum was evaluated as Absorption = Extinction - Reflection. The corresponding spectra for the array in Figure 4 are shown in Figure S1 for normal incidence of the excitation light.

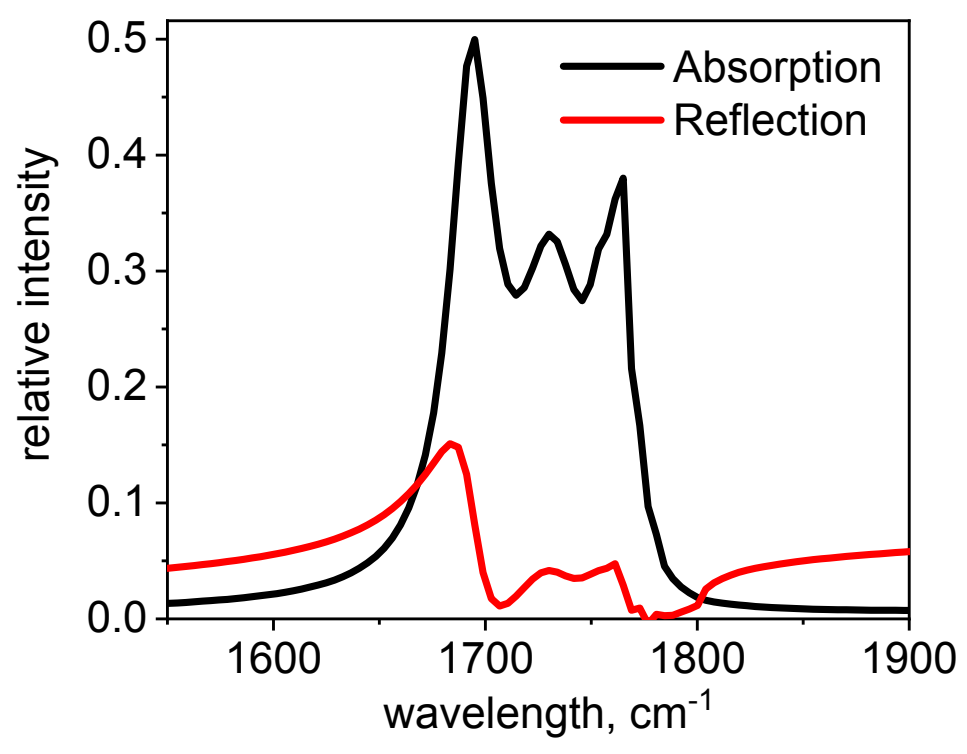


Figure S1. Calculated spectra of ALR array strongly coupled to the carbonyl stretching mode of PMMA. The parameters of the array are $\Lambda_{l}=1.4 \mu \mathrm{m}, \Lambda_{t}=4.15 \mu \mathrm{m}, \mathrm{L}=0.9 \mu \mathrm{m}, \mathrm{w}=0.2 \mu \mathrm{m}$. The thickness of the PMMA layer is $500 \mathrm{~nm}$. Normal incidence of the excitation light was used.

For the calculations of the near-field distribution at normal incidence, we used excitation with a plane wave source together with symmetric and anti-symmetric in-plane boundary condition.

\section{Evaluation of molecular fraction participating in the strong coupling}

In order to estimate the amount of molecules participating in the strong coupling with the ALR, we used the approach of ref. ${ }^{3}$ First, we calculated numerically the distribution of the electric field $E(r)$ within the unit cell of the array at the frequency of the ALR transition (omitting the resonant contribution of the vibrational transitions), and obtained the relevant field strength using normalization of the energy stored in the field to the expectation value of the field energy operator, ${ }^{4}$ according to

$$
\int \bar{W}(r) d V=\left\langle 0\left|\hbar \omega_{A L R}\left(\hat{a}^{\dagger} \hat{a}+\frac{1}{2}\right)\right| 0\right\rangle=\frac{\hbar \omega_{A L R}}{2}
$$

where $\bar{W}(r)$ is the energy density of the field at position $r,{ }^{5,6}$ given by

$$
\bar{W}(r)=\frac{\varepsilon_{0}}{4}\left(\frac{\varepsilon^{\prime}+2 \omega \varepsilon^{\prime \prime}}{\gamma_{e}}\right)|E(r)|^{2}
$$

where $\varepsilon_{0}$ is the permittivity of vacuum, $\varepsilon=\varepsilon^{\prime}+\varepsilon^{\prime \prime}$ is the permittivity of the material (in our case the permittivity of gold antenna and the non-resonant component of the permittivity of the PMMA polymer), $\gamma_{e}$ is the corresponding intrinsic dissipation rate, $\omega$ is the angular frequency of the excitation light (here, $\left.\omega=\omega_{A L R}\right), \hbar \omega_{A L R}$ is the energy of the open cavity mode at the ground state $|0\rangle$, and $\hat{a}^{\dagger}$ and $\hat{a}$ are the cavity rising and lowering operators.

Next, we calculated the coupling strength constant $g(r)=\mu \cdot E(r)$ between the ALR mode and the molecular transitions at each position $r$, assuming the PMMA monomer density of $\sim 1 \mathrm{~g} / \mathrm{cm}^{3}$ and the value of the vibrational transition dipole moment of $\mu=0.4$ Debye. The resulted total coupling strength between the molecular and ALR modes was evaluated as $g_{\text {tot }}=\sqrt{\sum g\left(r_{i}\right)^{2}}$, where $r_{i}$ are the discrete positions defined by the 3D mesh of our numerical simulations. We obtained $g_{\mathrm{tot}}=35 \mathrm{~cm}^{-1}$, which matches the value of half-the-splitting of the polariton mode energies obtained in the simulations. 
Finally, we examined the distribution of the coupling strength constants $g\left(r_{i}\right)$ illustrated in the histogram shown in Figure S2. The total population of $g\left(r_{i}\right)$ values can be divided into two fractions: $\sim 20 \%$ of the molecules with $g\left(r_{i}\right)>0.04 \mathrm{~cm}^{-1}$ and the remaining molecules with $g\left(r_{i}\right)<0.04 \mathrm{~cm}^{-1}$. Evaluating the effective coupling strength separately for each of the fractions, we found that $20 \%$ of molecules from the former fraction are responsible for most of the coupling, as $g_{20 \%}=\sqrt{\sum_{g>0.04 \mathrm{~cm}^{-1}} g\left(r_{i}\right)^{2}} \sim 30 \mathrm{~cm}^{-1}$, whereas the rest $80 \%$ of the molecules contribute only marginally. The relevant molecules, therefore, are located within the first 100 nanometers of the polymer layer.

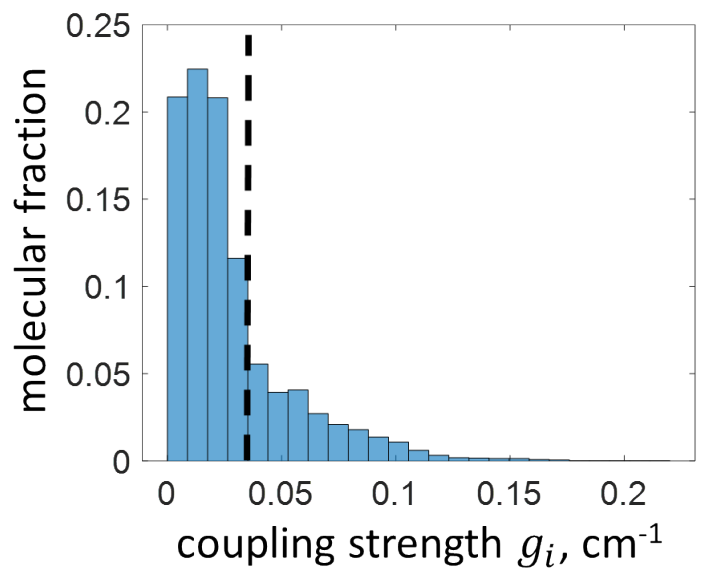

Figure S2. Distribution of the local coupling strength constants.

\section{References}

(1) Johnson, P. B.; Christy, R. W. Optical Constants of the Noble Metals. Phys. Rev. B 1972, 6, 43704379.

(2) Chen, K.; Adato, R.; Altug, H. Dual-Band Perfect Absorber for Multispectral Plasmon-Enhanced Infrared Spectroscopy. Acs Nano 2012, 6, 7998-8006.

(3) Gandman, A.; Mackin, R. T.; Cohn, B.; Rubtsov, I. V.; Chuntonov, L. Radiative Enhancement of Linear and Third-Order Vibrational Excitations by an Array of Infrared Plasmonic Antennas. Acs Nano 2018, 12, 4521-4528.

(4) Esteban, R.; Aizpurua, J.; Bryant, G. W. Strong Coupling of Single Emitters Interacting with Phononic Infrared Antennae. New J. Phys. 2014, 16, 013052.

(5) Ruppin, R. Electromagnetic Energy Density in a Dispersive and Absorptive Material. Physics Letters A 2002, 299, 309-312.

(6) Koenderink, A. F. On the Use of Purcell Factors for Plasmon Antennas. Opt. Lett. 2010, 35, 42084210. 
(7) Zheng, D.; Zhang, S.; Deng, Q.; Kang, M.; Nordlander, P.; Xu, H. Manipulating Coherent PlasmonExciton Interaction in a Single Silver Nanorod on Monolayer Wse2. Nano Lett. 2017, 17, 38093814. 